\title{
Caracterización y diferenciación de cafés, a partir de espectroscopía infrarroja
}

\section{Characterization and differentiation of coffee from infrared spectroscopy}

\author{
Óscar M. Barrera B. ; Nelson Gutiérrez G. ${ }^{2}$; Dayana Orozco-Blanco ${ }^{3}$
}

\begin{abstract}
1 Ing. Agrícola, M.Sc. Ciencia e ingeniería de alimentos, Grupo investigación agroindustria usco. Mild Coffee Company Huila S.A.S. Calle 9 No 8 - 60, Pitalito,
\end{abstract} Colombia, e-mail: osmabber@gmail.com, Dhttps://orcid.org/0000-0002-1837-046X

${ }^{2}$ Ing. Agrícola, Ph.D. Tecnología de alimentos, Grupo investigación agroindustria usco. Universidad Surcolombiana, Avenida Pastrana Borrero Carrera 1A, Neiva, Colombia, e-mail: ngutierrezg@usco.edu.co, Dhttps://orcid.org/0000-0003-2499-8066

${ }^{3}$ Ing. Agrícola, Grupo investigación agroindustria usco. Universidad Surcolombiana, Avenida Pastrana Borrero Carrera 1A, Neiva, Colombia, e-mail: dayana. orozco@hotmail.com, Dhttps://orcid.org/0000-0001-7523-407X

Cómo citar: Barrera B., Ó.M.; Gutiérrez G., N.; Orozco-Blanco, D. 2019. Caracterización y diferenciación de cafés, a partir de espectroscopía infrarroja. Rev. U.D.C.A Act. \& Div. Cient. 22(1):e1158. https://doi.org/10.31910/rudca.v22.n1.2019.1158

Artículo de acceso abierto publicado por Revista U.D.C.A Actualidad \& Divulgación Científica bajo una licencia Creative Commons CC BY-NC 4.0

Recibido: Mayo 24 de 2018

Aceptado: Febrero 4 de 2019

\section{RESUMEN}

El café es la segunda bebida más consumida en el mundo después del agua, en el que Colombia contribuye con el $9 \%$ de la producción, destacándose la calidad del café del departamento del Huila (cafés de altura); sin embargo, no hay suficiente evidencia sobre la incidencia de la altura en la calidad sensorial y composición química. En ese sentido, se buscó caracterizar y diferenciar cafés, mediante el análisis del espectro infrarrojo (FTIR) y evaluación sensorial. 62 muestras de cafés especiales fueron caracterizados, cosechados en diferentes altitudes, obtenidas en dos periodos de cosecha. Los espectros obtenidos permitieron encontrar diferenciación en los picos asociados a ácidos clorogénicos $\left(1600-1650 \mathrm{~cm}^{-1}\right)$, entre grano verde y tostado, aunque no se observaron diferencias en los picos, según el periodo de cosecha, por sí solos. La evaluación sensorial, según la metodología SCA, 2015, no generaron diferencias estadísticamente significativas entre periodos de cosecha y variedades evaluadas. Al considerar conjuntamente el análisis sensorial y el análisis del espectro infrarrojo, se presentaron diferencias estadísticamente significativas entre periodos de cosecha, atribuibles al contenido de cafeína y al puntaje total en taza. Los resultados encontrados muestran que no existe correlación entre la altura del cultivo de café y la calidad final de la bebida.

Palabras clave: altura; análisis sensorial; cafeína; ácidos clorogénicos.

\section{ABSTRACT}

Coffee is the second most consumed beverage in the world after water, the Colombian coffee production contributes with $9 \%$ of total world production and Colombian coffee is recognized by quality and mild coffee. Today, there is not enough evidence on the influence of altitude in sensory quality and coffee chemical composition. In this sense, we sought to characterize and differentiate coffees by infrared spectrum analysis (FTIR) and sensory evaluation. 62 specialty coffees samples were harvested at different altitudes, obtained in two harvest periods. The spectra obtained allowed finding differentiation in the peaks associated with chlorogenic acids $\left(1600-1650 \mathrm{~cm}^{-1}\right)$ between green and roasted coffee beans, although no differences were observed in the peaks according to the harvest period; by itself. 
The sensory evaluation, according to the SCA methodology, 2015 did not generate statistically significant differences between harvest periods and evaluated varieties. When jointly considering the sensory analysis and the infrared spectrum analysis, there were statistically significant differences between harvest periods, attributable to the caffeine content and the total cup score. The results found show that there is no correlation between the height of the coffee crop and the final quality of the beverage.

Keywords: altitude; sensory analysis; chlorogenics acids; caffeine.

\section{INTRODUCCIÓN}

El café, además de ser una bebida popular, es el producto agrícola más consumido del mundo, dadas sus características organolépticas, debido al creciente consumo de cafés especiales y a los diferentes métodos de preparación (Alves et al. 2010; Cheong et al. 2013; Di Bella et al. 2014). De acuerdo con la Organización Internacional del Café, el consumo de café, a partir del 2012, presentó una tasa media de crecimiento anual del $2 \%$, liderada por Asia y Oceanía, seguidos de Norteamérica y África; por debajo de la media crecieron Europa, Sudamérica y América Central (OIC, 2016). Colombia, se destaca por la producción de cafés suaves y de alta calidad. En la actualidad, es el mayor productor de cafés arábigos lavados y el tercero en la producción mundial; en la región del Huila, se producen los más altos volúmenes de café, con una participación de 2 millones 560 mil sacos de café verde año, correspondientes al 18\% de la producción nacional (Dávila, 2018).

En la última década, los cafés producidos en la región Huila obtuvieron reconocimiento internacional, por los diferentes premios obtenidos en los concursos de calidad en taza. Esta calidad, se atribuye a la consistencia en sus atributos, asociada a las variedades cultivadas, el manejo agronómico, los procesos poscosecha y las condiciones agrometeorológicas del cultivo; algunos estudios relacionan la influencia de la altura sobre la calidad final en taza (Suárez et al. 2015), mientras que otros autores no reportan relación directa entre ellas (Orozco et al. 2011). La calidad del café depende de muchos factores; sin embargo, la tostión se puede considerar como el parámetro más importante en el resultado final en la bebida (Wei \& Tanokura, 2015; Santos et al. 2016), el cual, promueve el desarrollo de componentes volátiles, como el aroma, atributo característico del café, que se forma durante este proceso, aunque las composiciones varían para los granos de café de diferentes especies, orígenes, grados de tostado o métodos analíticos. Se puede considerar que la formación del aroma característico, sabor y color resulta, primero, de la disminución drástica de los componentes del café verde, como la sacarosa, aminoácidos libres, ácidos clorogénicos (ACG) y trigonelina, así como de la descomposición de polisacáridos y proteínas (Wei \& Tanokura, 2015). Durante el proceso de tostión, los granos verdes sometidos a altas temperaturas de tostado causan una serie de cambios físicos y químicos en los granos. Las condiciones específicas de tostión influyen fuertemente, afectando la bioactividad y el sabor de la bebida (Farah, 2012).
La cafeína es uno de los componentes más importantes, ya que contribuye en el amargor y en el efecto estimulante de la bebida; si es consumida moderadamente puede tener beneficios, como el aumento de disponibilidad de energía, concentración y disminución de la fatiga (Cheng et al. 2016). En ese sentido, el café arábica es más popular por su bajo contenido de cafeína en comparación con el Robusta, con valores entre 0,9 - 1,3 y 1,5 - 2,5 (g/100g), respectivamente (Farah, 2012), teniendo mayor aceptación por los consumidores.

Los atributos acidez, cuerpo y amargura están directamente relacionado con el proceso de tostión, por tanto, un café especial, se caracteriza por el equilibrio de estos atributos (Melo, 2004). Los ACG son los responsables de contribuir en la amargura de la bebida durante el proceso de tostión, ya que son drásticamente degradados y su percepción es baja sensorialmente. La clase de lactonas que descomponen el producto de ácidos clorogénicos ha sido identificada como uno de los principales contribuyentes al amargor en el café. Por otro lado, la cafeína presente en el café verde $10-20 \%$ y otorga un fuerte sabor amargo (Schenker \& Rothgeb, 2017).

Recientes investigaciones sugieren la implementación del uso de métodos de espectroscopia infrarroja FTIR (Fourier Transform Infrared, por sus siglas en inglés), en la caracterización y evaluación de la calidad en alimentos; de acuerdo con Rodriguez \& Allendorf (2011) y Cebi et al. (2017), esta metodología es confiable, rápida, reproducible, fácil de realizar, no requiere preparación de muestras y no destructiva. El análisis del espectro infrarrojo se ha utilizado con éxito para encontrar diferencias entre granos defectuosos y no defectuosos de café almendra (Craig et al. 2012). Otros estudios, se han desarrollado para determinar diferencias en granos de café procesados con diferentes protocolos de tostión (Wang \& Lim, 2012); admás, se detectaron adulteraciones de café tostado y molido, mezclado con maíz y cebada (Reis et al. 2013).

En este estudio, se utilizó la técnica FTIR-ATR (Fernandes $e t$ al. 2014), para encontrar diferencias entre muestras de café recolectadas en dos periodos diferentes de cosecha; además, se realizó un análisis estadístico multivariable, incluyendo los atributos sensoriales, la altura de la plantación y los resultados del espectro, para buscar una tendencia que definiera diferencias en función de la altura y la calidad final en taza.

\section{MATERIALES Y MÉTODOS}

Se evaluaron 62 muestras de granos de café de las variedades Caturra (C) y Castillo (K), cultivadas entre 1.200 y $2.000 \mathrm{~m}$ s.n.m., en cuatro municipios del sur del Huila: Acevedo, Pitalito, San Agustín e Isnos (Colombia), para dos periodos de cosecha: mitaca (abril, mayo y junio) y principal (octubre, noviembre y diciembre) (Henao et al. 2017). El beneficio, se realizó en las mismas condiciones para todas las muestras, con un tiempo de fermentación de 19 horas; el proceso de secado, se llevó hasta obtener un contenido de humedad del grano entre $10-12 \%$. Las muestras, como café pergamino seco, fueron 
transportadas hasta el Centro Surcolombiano de Investigación en Café (CESURCAFÉ), de la Universidad Surcolombiana. La etapa del proceso de beneficio, se desarrolló en el Tecnoparque Yamboró, en Pitalito.

Preparación de las muestras: Se obtuvieron muestras por triplicado de café almendra verde después de trillar $500 \mathrm{~g}$ de café pergamino seco por 1 minuto, en la trilladora de muestras INGESEC; la almendra, se tamizó sobre la malla \# 13 ING-Z-TAMIZ-E de INGESEC, de acuerdo con la norma NTC5248 y se realizó la selección de almendra sana (SCAA, 2015); submuestras de $150 \mathrm{~g}$ de almendra sana fueron tostados en una tostadora de muestras PROBAT, de acuerdo con el protocolo de la SCAA (2015), con una temperatura inicial de $200^{\circ} \mathrm{C}$.

Análisis fisicoquímico: El contenido de humedad, se determinó mediante el método dieléctrico (capacitancia), con el medidor de humedad KETT PM-450 (Kett electric laboratory, Santiago Boulevard); la prueba, se realizó por triplicado. Los granos de café enteros verdes y tostados fueron medidos en el espacio CIEL $* a * b *$, directamente en la celda de $50 \mathrm{~mm}$ (diámetro) e iluminante $\mathrm{D}_{65}$, previamente calibrados con los blancos del equipo y, las mediciones, se realizaron por triplicado, con el Colorímetro Konica Minolta CR410. Las mediciones del infrarrojo, se efectuaron en un espectrómetro FTIR SISTEMA AGILENT Cary 630-FTIR accesorio ATR, con longitudes de onda de $4000-700 \mathrm{~cm}^{-1}$; para café verde, los espectros se registraron en longitudes de onda de $4000-700 \mathrm{~cm}^{-1}$, con $8 \mathrm{~cm}^{-1}$ de resolución, 8 escáneres y, para café tostado, los espectros se registraron en una longitud de onda de $3500-1000 \mathrm{~cm}^{-1}$, con $8 \mathrm{~cm}^{-1}$ de resolución, 8 escáneres.

Análisis sensorial: Las muestras fueron evaluadas de acuerdo con la metodología de la Asociación de Cafés Especiales - SCA, por sus siglas en inglés (SCAA, 2015), en diferentes fases: Preparación, las muestras de café verde fueron sometidas al proceso de tostión, con temperatura inicial de $200^{\circ} \mathrm{C}$; finalizado son molidas y dosificadas, en una relación peso/volumen, de 8,25 gr (+/- 0,25 gr) de café, para $150 \mathrm{ml}$ de agua por taza. Evaluación de la bebida, en esta fase, se valoraron 10 atributos de la bebida (fragancia/aroma, sabor, sabor residual, cuerpo, acidez, uniformidad, balance, limpieza de taza, dulzor y valoración final del catador), para cada muestra, reportados en el formato de evaluación diseñado por la SCA; finalmente, el panel de evaluación estuvo conformado por 5 catadores entrenados.

Análisis estadístico: Se aplicaron pruebas de estadística multivariable, análisis de clúster (conglomerado), con el objetivo de agrupar variables, tratando de lograr la máxima homogeneidad en cada grupo y análisis de componentes principales (ACP), para los resultados sensoriales, en conjunto con los análisis fisicoquímicos. Se utilizó el software estadístico StatGraphics Centurion XV.II para Windows.

\section{RESULTADOS Y DISCUSIÓN}

En las figuras 1 y 2, se presentan los espectros en muestras de café para los estados de almendra verde y tostado, para los dos periodos cosecha evaluados (mitaca y principal); atendiendo lo reportado por Ribeiro et al. (2010), para muestras de café en grano la longitud de onda de $1750 \mathrm{~cm}^{-1}$, está asociada a la presencia de cafeína (C) y los picos obtenidos entre $1600-1650 \mathrm{~cm}^{-1}$, se asocian a ácidos clorogénicos (ACG). En la figura 1, se observa que, tanto para la C como para los ACG, los picos son más definidos en comparación a la figura 2 , en la que los picos asociados a los ACG $\left(1600\right.$ y $\left.1650 \mathrm{~cm}^{-1}\right)$ aparecen disgregados, debido a que estos ácidos son térmicamente inestables y su pérdida en café arábica, después del proceso de tostado, puede llegar a ser superior al 95\%. Esta disminución, se debe a que los ACG se degradan, debido a la ruptura de los enlaces carbono-carbono (Farah et al. 2005), para formar ácido cafeico, lectonas y otros derivados del fenol, a través de las reacciones de Maillard y Strecker, lo que se traduce en un aumento en el amargor, astringencia y aroma (Gotteland \& De Pablo, 2007; Cheng et al. 2016). Para el caso de la cafeína, no presenta cambios significativos, debido a que, según Oestreich-Janzen (2013), es un compuesto nitrogenado que no se ve afectado por el proceso de tostado; sin embargo, otro factor que puede estar asociado en el contenido de estos compuestos es la altitud. Según Worku et al. (2018), en su estudio, estableció que estos compuestos fueron afectados por la altitud, un aumento de 100m de altura, los contenidos de C y ACG disminuyen significativamente.

En la figura 3, se presenta el ACP para las muestras conjuntas de cosecha principal y mitaca, incluyendo, como variables, los resultados del análisis sensorial; se consideró además, como observación, las alturas de las plantaciones clasificadas como rango de altura baja $1.200-1.500 \mathrm{~m}$ s.n.m., media $1.500-1.750 \mathrm{~m}$ s.n.m. y alta $1.750-2.000 \mathrm{~m}$ s.n.m.; se puede observar una dispersión general de las muestras en ausencia de un modelo de separación definido, lo cual, confirma lo reportado por Orozco et al. (2011), quienes indican que la altura no es un factor determinante sobre la calidad final en taza.

El análisis conjunto de la altura de las plantaciones, con los atributos sensoriales y la cafeína, no generaron algún patrón distinto a una simple dispersión de las muestras, mientras que al involucrar en las observaciones el periodo de cosecha, se generó un patrón de clasificación, que permitió la construcción de tres conglomerados perfectamente diferenciados, como se muestra en la figura 4 (método de promedio de grupo eclidiana). Este patrón puede estar relacionado con las diferencias que se generan en las interacciones obtenidas entre los picos de cafeína y el puntaje total en taza que, en las muestras del conglomerado 1 , correspondiente a la cosecha principal, tenían menor área en los picos de cafeína $(1,29 \pm 0,32)$ y menor puntaje en taza $(82,99 \pm 0,37)$, mientras que el conglomerado 2 , también obtenido en cosecha principal, tienen área de cafeína y puntaje en taza más altos, con un promedio de $2,05 \pm 0,41$ y $84,90 \pm 0,48$, respectivamente; para el conglomerado 3 , el área de los picos de cafeína fue de 1,32 $\pm 0,27$ y el puntaje final en taza fue de $84,85 \pm 0,9$; se puede inferir que esta diferencia esté asociada a condiciones climáticas presentadas durante los periodos de cosechas evaluadas. Estos resultados son similares a los reportados por Worku et al. (2018), quienes encontraron, en dos años de estudio, en diferentes cosechas, variaciones en los contenidos de C y ACG. Esto se puede explicar por la relación entre: ubicación del cultivo, condiciones de crecimiento, composición bioquímica de los granos de café y altitud. 


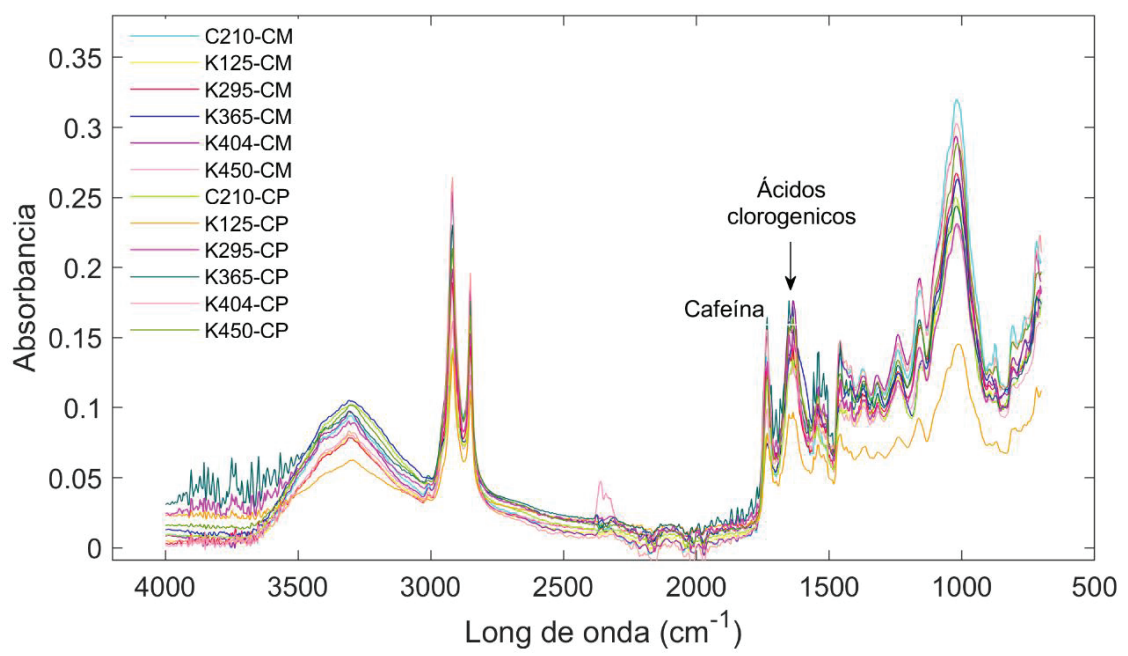

Figura 1. Espectro de muestras de café verde de las cosechas (CM: cosecha mitaca y CP: cosecha principal).

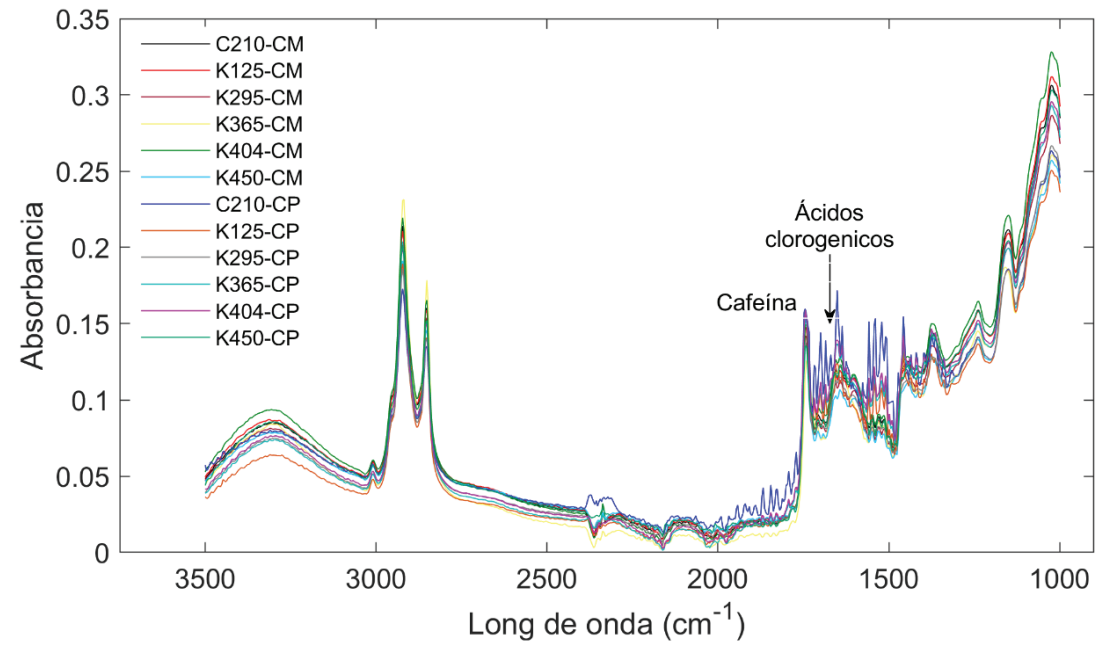

Figura 2. Espectro de muestras de café tostado de las cosechas (CM: cosecha mitaca y CP: Cosecha principal), de las variedades castillo (K) y caturra (C).
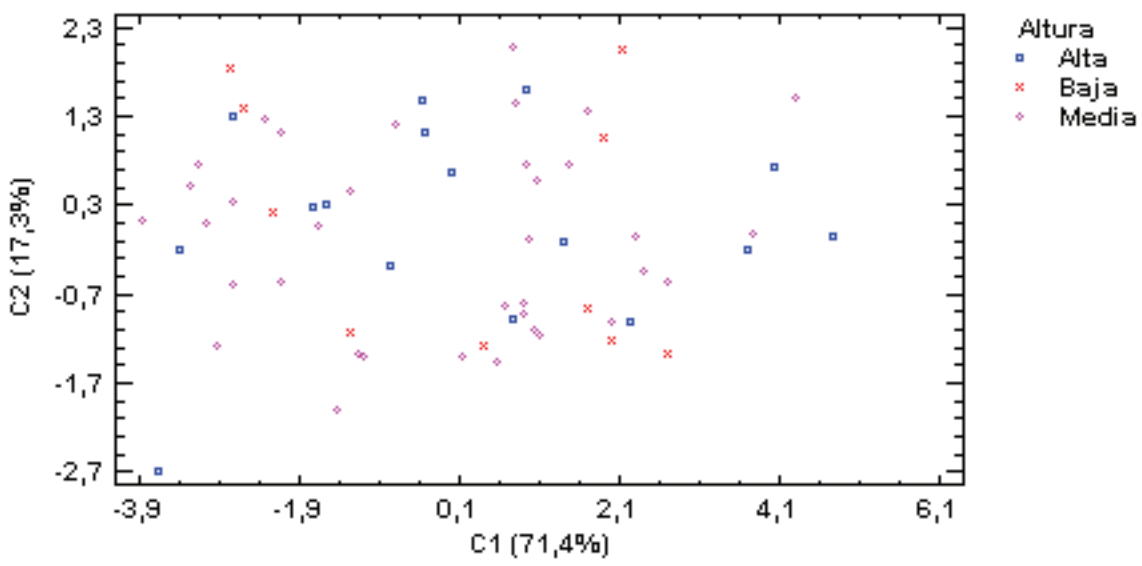

Figura 3. Análisis de componentes principales de evaluación sensorial en función a la altura sobre el nivel del mar del cultivo. 


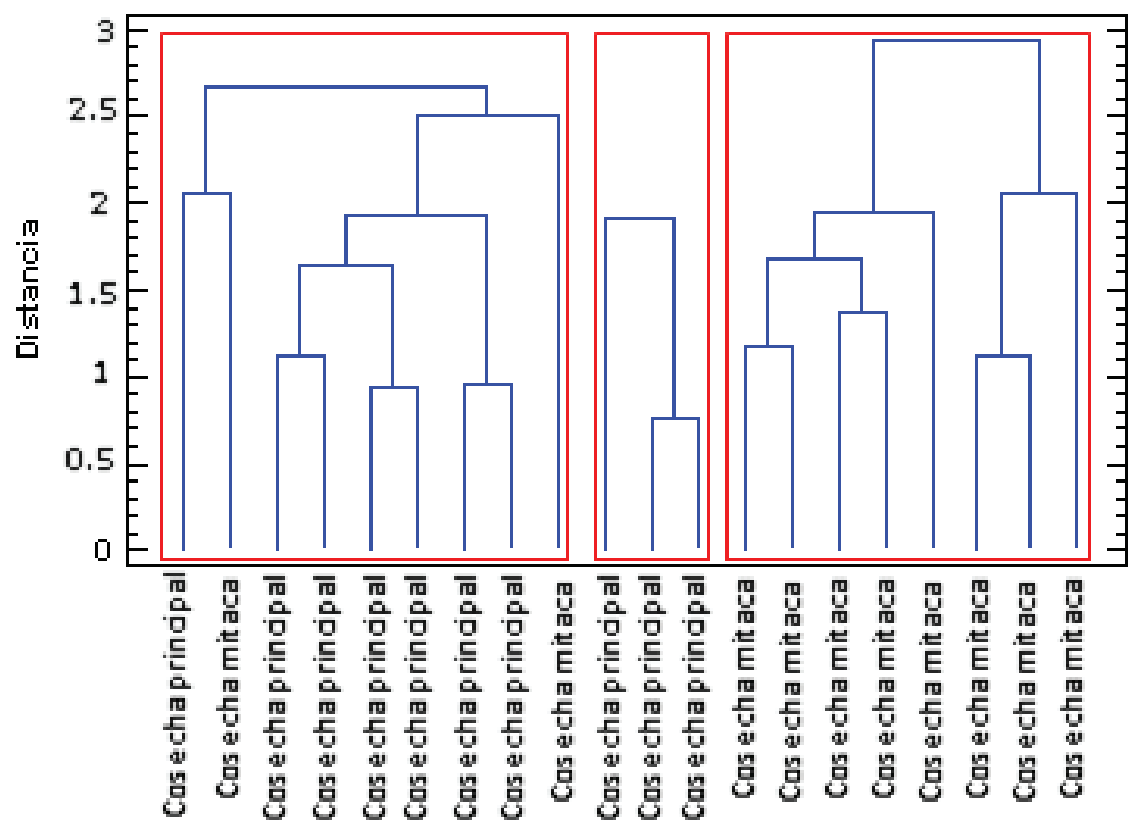

Figura 4. Dendograma. Método de promedio de grupo, Euclideana, entre las muestras recolectadas en dos periodos de recolección (cosechas): pincipal y mitaca.

Por otro lado, De Lima et al. (2015) establecen que la interacción entre el genotipo del cultivo y el ambiente es compleja y afecta directamente la calidad de la bebida, que se constituye de atributos importantes, como: físicos, organolépticos y compuestos químicos (C, ACG y trigonelina); cada uno de éstos atributos depende de nivel de sombrío, temperatura diaria, cantidad y distribución de la lluvia (Debela \& Vos, 2017).

Los análisis de los espectros en muestras de café en grano, obtenidos con la técnica FTIR-ATR, permitieron observar diferencias en los picos asociados a los ácidos clorogénicos para muestras recolectadas en diferentes periodos de tiempo; sin embargo, es necesario implementar técnicas cromatográficas, que permitan detectar, identificar y cuantificar los compuestos de interés.

Analizados individualmente, los resultados de la evaluación sensorial con la metodología SCAA, no permiten determinar una relación directa entre las alturas de siembra del cultivo de café con la calidad final en taza.

En el estudio, se determinó que no existe correlación entre la altura del cultivo de café y de las variedades sobre la calidad final de la bebida, al igual que entre periodos de cosecha (mitaca/principal). En el proceso de postcosecha, los parámetros ambientales, como temperatura, humedad relativa, estado de madurez, cantidad y distribución hídrica en el desarrollo del fruto, están relacionados directamente con la calidad final. Es recomendable utilizar análisis descriptivo cuantitativo (QDA) con los mismos atributos que incluye la metodología SCA, abarcando la valoración de la intensidad del atributo y de las notas resultantes en la evaluación; además, tener en cuenta otros aspectos, como el grado de sombrío del cultivo y genotipo

Conflictos de intereses: Este artículo fue preparado y revisado con la participación de todos los autores, quienes declaramos que no existe conflicto de intereses que ponga en riesgo la validez de los resultados presentados. Financiación: Este estudio fue financiado por el proyecto de Investigación: Determinación de Huellas Ambientales como Indicador en la Toma de Decisiones en el Manejo Integral de la Producción de Cafés Especiales en el Sur del Departamento del Huila.

\section{REFERENCIAS}

1. ALVES, R.C.; CASAL, S.; OLIVEIRA, M. 2010. Tocopherols in coffee brews: Influence of coffee species, roast degree and brewing procedure. J. Food Compos. Anal (United States). 23(8):802-808. https://doi.org/10.1016/j.jfca.2010.02.009

2. CEBI, N.; TAHSIN, Y.M.; SAGDIC, O. 2017. A rapid ATR-FTIR spectroscopic method for detection of sibutramine adulteration in tea and coffee based on hierarchical cluster and principal component analyses. Food Chem (England). 229(15):517-526. https://doi. org/10.1016/j.foodchem.2017.02.072

3. CHEONG, M.W.; TONG, K.H.; MING O., J.J.; LIU S., Q.; CURRAN, P.; YU, B. 2013. Volatile composition and 
antioxidant capacity of Arabica coffee, Food Res. Int. (Canada) 51(1):388-396. http://dx.doi.org/10.1016/j. foodres.2012.12.058

4. CHENG, B.; FURTADO, A.; SMYTH, H.E.; HENRY, R.J. 2016. Influence of genotype and environment on coffee quality, Trends Food Sci Technol (England). 57:20-30. http://dx.doi.org/10.1016/j.tifs.2016.09.003

5. CRAIG, A.P.; FRANCA, A.S.; OLIVEIRA, L.S. 2012. Evaluation of the potential of FTIR and chemometrics for separation between defective and non-defective coffees. Food Chem. 132(3):1368-1374. http://dx.doi. org/10.1016/j.foodchem.2011.11.121

6. DÁVILA, L. 2018. Así fue la cosecha cafetera del Huila en 2017. http://www.lanacion.com.co/2018/01/05/asi-fuela-cosecha-cafetera-del-huila-2017/

7. DEBELA, B.A.; VOS, J. 2017. Tree management and envionmental conditions affect coffee (Coffea arabica L.) bean quality. NJAS-WAGEN J LIFE SC. (Netherlands) 83:39-46. https://doi.org/10.1016/j.njas.2017.09.002

8. DI BELLA, G.; POTORTİ, A.G.; LO TURCO, V.; SAITTA, M.; DUGO, G. 2014. Plasticizer residues by HRGC-MS in espresso coffees from capsules, pods and moka pots, Food Control (England) 41:185-192

9. DE LIMA, A.E.; GUIMARAES, M.A.N.; RODRIGUES, C.G.; BOTELHO, C.E.; DE MELO, C.E.; CARDOSO, D. 2015. Desempenho agronômico de populações de cafeeiros do grupo ‘Bourbon’. Coffee Science. (Brazil) 11: 22-32. http:// dx.doi.org/10.25186/cs.v11i1.957

10. FARAH, A.; PAULIS, T.; TRUGO, C.L.; MARTIN, P.R. 2005. Effect of roasting on the formation of chlorogenic acid lactones in coffee. J. Agric. Food Chem. (United States). 53(5):1505-1513. https://doi.org/10.1021/jf048701t

11. FARAH, A. 2012. Coffee Constituents, in Coffee: Emerging Health Effects and Disease Prevention (ed Y.-F. Chu), Wiley-Blackwell, Oxford, UK. https://doi. org/10.1002/9781119949893.ch2

12. FERNANDES, B. D.; MADUREIRA, F. A. L.; SUN, D. W.; NIXDORF, S. L.; YOKO H. 2014. Application of ingrared spectral techniques on quality and compositional attributes of coffee: an overview. Food Res Int. (United States). 61:23-32. https://doi.org/10.1016/j.foodres.2014.01.005

13. GOTTELAND, M.; DE PABLO, V.S. 2007. Algunas verdades sobre el café. Rev. chilena de nutrición. 34(2):105-115. http://dx.doi.org/10.4067/S0717-75182007000200002
14. HENAO, J.D.; GUTIÉRREZ, G.N.; MEDINA R., D.R. 2017. Buenas prácticas y procedimientos para el acopio de cafés especiales. Editorial Universidad Surcolombiana. 80p.

15. MELO, W.L.B.A. 2004. Importância da informação sobre do grau de torra do café e sua influência nas características organolépticas da bebida. São Carlos: Empresa Brasileira de Pesquisa Agropecuária (Embrapa). Comunicado Técnico 58. $4 \mathrm{p}$.

16. ORGANIZACIÓN INTERNACIONAL DE CAFÉ, OIC. 2016. Informe del mercado del café junio 2016. El consumo de café aumenta y los precios en el mercado son los más altos en 14 meses. Disponible desde internet en: http:// www.ico.org/documents/cy2015-16/cmr-0616-c.pdf [Con acceso el 9/04/2018]

17. OESTREICH-JANZEN, S. 2013. Chemistry of Coffee. In: Reedijk, J. (Ed.) Elsevier Reference Module in Chemistry, Molecular Sciences and Chemical Engineering. Waltham, MA: Elsevier. https://doi.org/10.1016/B978-0-12-4095472.02786-4

18. OROZCO, C.N.; GUACAS, S.A.; BACCA, T. 2011. Caracterización de fincas cafeteras por calidad de la bebida y algunas condiciones ambientales y agronómicas. Rev. Ciencias Agrícolas. 28(2):9-17.

19. REIS, N.; FRANCA, A.S.; OLIVEIRA, L.S. 2013. Discrimination between roasted coffee, roasted corn and coffee husks by Diffuse Reflectance Infrared Fourier Transform Spectroscopy. Food Sci. Technol. (Brasil). 50(2):715-722. https://doi.org/10.1016/j.lwt.2012.07.016

20. RIBEIRO JULIANO, S.; SALVA TEREZINHA, J.; FERREIRA MÁRCIA, M.C. 2010. Chemometric studies for quality control of processed brazilian coffees using drifts. J. Food Quality. 33:212-227. https://doi.org/10.1111/j.17454557.2010.00309.x

21. RODRIGUEZ-SAONA, L.; ALLENDORF, M.E. 2011. Use of FTIR for rapid authentication and detection of adulteration of food. Annu. Rev Food Sci Technol (United States). 2:467-483. https://doi.org/10.1146/annurevfood-022510-133750

22. SANTOS, J.R.; VIEGAS, O.; PÁSCOA R., N.M.J.; FERREIRA I., M.P.L.V.O.; RANGEL A., O.S.S.; LOPES, J.A. 2016. Inline monitoring of the coffee roasting process with near infrared spectroscopy: Measurement of sucrose and colour. Food Chem. 208:103-110. https://doi.org/10.1016/j. foodchem.2016.03.114 
23. SPECIALTY COFFEE ASSOCIATION OF AMERICA, SCAA. 2015. SCAA Protocols: cupping specialty coffee. Disponible en internet en: http://scaa. org $/$ ?page $=$ resources $\& d=$ cupping-protocols $[$ con acceso el 13/06/2016]

24. SCHENKER, S.; ROTHGEB, T. 2017. The roast creating the beans' signature. In the craft and science of coffee. Ed. Britta Folmer (London): p.245-271. https://doi. org/10.1016/B978-0-12-803520-7.00011-6

25. SUÁREZ, S.J.; RODRÍGUEZ, B.E.; DURAN, B.E. 2015. Efecto de las condiciones de cultivo, las características químicas del suelo y el manejo de grano en los atributos sensoriales de café (Coffea arabica L.) en taza. Acta Agronómica. 64(4):342348. http://dx.doi.org/10.15446/acag.v64n4.44641_
26. WANG, N.; LIM, L.T. 2012. Fourier transform infrared and physicochemical analyses of roasted coffee. J. Agric. Food Chem. 60(21):5446-5453. https://doi.org/10.1021/ jf300348e

27. WEI, F.; TANOKURA, M. 2015. Chapter 10 - Chemical Changes in the Components of Coffee Beans during Roasting, In Coffee in Health and Disease Prevention, edited by Victor R. Preedy, Academic Press: p.83-91.

28. WORKU, M.; MEULENAER, B.; DUCHATEAU, L.; BOECKX, P. 2018. Effect of altitude on biochemical composition and quality of green Arabica coffee beans can be affected by shade and postharvest processing method. Food Res. Internal. (United States) 108:278-285. https:// doi.org/10.1016/j.foodres.2017.11.016 